\title{
Personal Health Data: Access and Perceived Value in Denmark
}

\author{
M. Karampela, C. Grundstrom, and M. Isomursu
}

\begin{abstract}
This study explores how accessible and valuable Personal Health Data are in Denmark. This paper uses a qualitative inquiry which was adopted to provide information about (1) the accessibility of data available, (2) and the perceived value of data by recruiting 8 healthy Danish individuals who were instructed to access their personal health data, and were then prompted to discuss how accessible and valuable they perceived their personal health data to be. In total, participants accessed 31 datasets and wearable sensor data through 23 web applications and 8 mobile applications. They reported on search and access challenges in interviews and through journaling. Our results suggest that participants were satisfied with the access they had to their personal health data, however the participants expressed disappointment in ways the data was presented for them by the services and platforms. Thus, we concluded that the perceived value of personal data were found to be dependent on the usability and personalization features of the services, rather than on the data itself.
\end{abstract}

\section{INTRODUCTION}

In modern society, digital traces of everyday life are scattered across world-wide information networks and stored in various databases. The growing amount of online personal data that are available to everyone, consist of data stored actively by individuals but also by public or private organizations on behalf of them. It is hard to estimate the amount of personal digital data or digital footprint that individuals develop in everyday life [1]. The digital footprint can be informative about individuals' behaviors, relationship patterns and preferences [2,3,4]. For example, the Google Now app and Siri, are applications that utilize user's digital footprint to personalize services based on predictive analytics $[5,6]$.

Previous research addressed the potential benefits of the use of digital footprint to improve the management of lifestyle related diseases [7]. Even so, individuals are hesitant to actively collect and use their digital footprint, due to obstacles related to the format and interoperability of the data and poor user interface design [8]. Accessibility of digital health records can facilitate effective communication between clinicians and patients, fostering the emergence for innovative health services $[9,10]$. Despite the fact that the potential value of adopting electronic health records (EHRs) for self-care and wellbeing has been addressed, research regarding the accessibility of data stored in various public and private organizations is still in its early stages.

M.K. and M.I. are with the Digital Design Department, IT-University of Copenhagen, Rued Langgaards Vej 7, DK-2300 Copenhagen S, Denmark (phone: (+45)721-850-00; e-mail: makar@itu.dk, miis@itu.dk).

C. G. and M.I. are with the Interact Group, University of Oulu P.O. Box 8000, Finland (e-mail: casandra.grundstrom@oulu.fi, minna.isomursu@oulu.fi).
This study builds upon the studies of [11,12] by exploring the accessibility and perceived value of health-related digital footprints, by expanding the investigation originally from Finland to the Danish context. We aim to explore the availability of personal health data for health selfmanagement. Nordic countries have high level of digitalization, and have invested a lot in digital health registries, which leads us to assume that the availability of Personal Health Data (PHD) in Finland and Denmark would be favorable. The present study addresses the following research question: In Denmark, what are the accessibility challenges of personal health data, and what is the perceived value of personal health data available?

The definition used in this paper for perceived value is based on the participants experience and needs when accessing their PHD through a service or platform. The service or platform has inherent properties which are contextual to the participants' interactions where the perceived value is derived [13]. The role of accessibility in this experience occurs when a participant needs to have a healthcare opportunity fulfilled, such as: viewing, retrieving, or interacting with their PHD which is provided by a service or platform thus affording means and opportunity [14].

In Denmark, the independent agency Datatilsynet, enacts the law for data accessing, processing and protection, in which it is expressly stated residents' right of access to their personal data that are stored in databases of organizations [15]. Thus, according to this legislation, our participants were anticipated to be able to access their personal health digital footprint. Our results indicate the feasibility of access to PHD in Denmark, as well as the perceived value of the data based on the subjective experience of the participants. We believe this knowledge provides a baseline for future research towards the creation of innovative health services.

The paper proceeds as follows: firstly, we explain the methodology for collecting the data, then detail the results together with the discussion, and finally, the conclusion with the limitations and directions for future research.

\section{METHODOLOGY}

A group of 12 participants (3 natives, 9 non-natives) were asked via email to participate in the study. They were instructed to access any data source that could help them to interpret their own health, health habits and behavior. As the study progressed, 4 of the participants chose not to continue completing all data collection tasks, and are therefore left out from the analysis. To facilitate data collection, researchers shared a list of potential organizations with participants, although they were instructed to decide themselves which data sources could be valuable for them. In order to keep track of accessed data, participants were provided with a 
Word document, and were instructed to store the names of organizations they accessed.

Using a semi-structured interview guide, 6 of the participants were interviewed face-to-face and two via Skype. The interview guide was comprised of two themes; experiences of accessing data and data usage. Researchers were interested in capturing the experiences and challenges of participants while accessing their PHD, personal expectations and finally information accuracy. In addition, participants were encouraged to share their personal opinion regarding future data usage and how valuable they thought their personal information to be. Before the study all the participants received and signed an informed consent form.

In total 8 healthy participants, all residing in Denmark for more than 2 years, agreed to voluntarily participate in this study. The 8 active participants ( 2 males and 6 females) over a period of 3 weeks accessed 31 databases and wearable sensor data (23 through web applications, 8 through mobile apps). Based on their nature, these sources were sorted into 14 subcategories, through a coding process resulting in the categories presented in Table 1.

TABLE I. SUMmary OF All SOURCES ACCESSED By PARTICIPANTS

\begin{tabular}{|c|l|c|l|}
\hline $\begin{array}{c}\text { Source } \\
\text { Categories }\end{array}$ & $\begin{array}{c}\text { Source } \\
\text { Services or } \\
\text { Platforms }\end{array}$ & $\begin{array}{c}\text { Source } \\
\text { Categories }\end{array}$ & $\begin{array}{c}\text { Source } \\
\text { Services or } \\
\text { Platforms }\end{array}$ \\
\hline Apps: & $\begin{array}{l}\text { 8Fit, BedTime, } \\
\text { Headspace, } \\
\text { Health iPhone, } \\
\text { Lifesum, } \\
\text { MyFitnessPal, } \\
\text { Yoga Studio, } \\
\text { Yummly }\end{array}$ & Grocery Stores: & Coop, Føtex \\
\hline $\begin{array}{c}\text { Beauty \& } \\
\text { Health Store }\end{array}$ & Matas & $\begin{array}{c}\text { Healthcare } \\
\text { Systems: }\end{array}$ & $\begin{array}{l}\text { Borger, } \\
\text { Care4U, } \\
\text { Sundhed }\end{array}$ \\
\hline Dentist: & $\begin{array}{l}\text { Plus1 } \\
\text { Tandlæger }\end{array}$ & Optical Stores: & $\begin{array}{l}\text { Louis Nielsen, } \\
\text { Synoptik }\end{array}$ \\
\hline Employers: & Inspiration & Social Media: & $\begin{array}{l}\text { Facebook, } \\
\text { Instagram }\end{array}$ \\
\hline Education: & $\begin{array}{l}\text { Københavns } \\
\text { Universitet }\end{array}$ & Transportation: & Rejseplanen \\
\hline Finance: & Nordea Bank & Wearables: & $\begin{array}{l}\text { Garmin, } \\
\text { Withings }\end{array}$ \\
\hline Fitness Chains: & $\begin{array}{l}\text { FitnessWorld, } \\
\text { FysiskForm }\end{array}$ & Web Apps: & $\begin{array}{l}\text { Amazon, } \\
\text { Apple, Google, } \\
\text { YouTube }\end{array}$ \\
\hline
\end{tabular}

Participants' interviews were transcribed together with their notes which were journaled in a Word document. These were analyzed thematically based on the themes in the interview guide. To analyze the data the following process was adopted; initially two researchers read participants' notes and read transcriptions to identify themes and subthemes that emerged from the research questions. All relevant quotes were then organized in subthemes, and researchers had three sessions in which preliminary results were discussed and themes and subthemes were redefined. Subsequently, a third researcher was included in the data analysis process. After an introduction to preliminary results, the researchers had two sessions in which they discussed the outline of themes and subthemes to arrive at the final results. Following this collaborative process, data were clustered into subthemes and results were analyzed. The analysis of the results focused on
(1) the participants' experiences while accessing their data (2) and the perceived value of data.

\section{RESULTS}

In this section, we will present the results in the following five categories. Quotes that support these categories can be found in Table 2 below.

\section{A. Access Protection}

When participants accessed their personal data, they reported the need for more fastidious security. Thus, illustrating that data access security verification methods were not always transparent or secure enough, and had participants reflecting on the safety procedures of accessing their PHD as seen in Quote 1. Additionally, security concerns arose when considering external sources (such as third parties) accessing their PHD. However, to mitigate their security concerns participants expressed desire to manage access to their personal data but not without some form of control over their personal data as expressed in Quote 2.

\section{B. Accessibility Challenges}

Accessibility challenges were experienced by all of the participants concerning poor user interface design. Several participants had a negative experience when navigating through the National Health Portal Sundhed, as they found broken links, incomplete pages, and a general lack of intuitive content and function, Quote 3 illustrates one of these challenges. The majority of the participants described the national health portal as a passive database that runs in the background which simply displayed personal information in a static form. The services or products that the participants were more engaged in for everyday life were considered to be more valuable sources of information for understanding their personal health. Participants reported that they felt they can access most of their personal information through online tools.

An additional challenge for the participants was related to uncertainty on where to find their PHD. The majority of the participants reported that it took some effort for them to identify sources that could be meaningful in describing their health, and then where to find the corresponding personal data. Many participants needed to brainstorm or use Google to help guess where to find their personal data as stated in Quote 4.

\section{Perceived Value of Accessed Personal Data}

Language barriers were experienced by all of the participants in regards to medical terminology. Several of the participants noted that medical language or jargon was used in their personal data and found it confusing. This was a barrier that was most commonly reported in regards to data accessed through Sundhed. The participants had no means to interpret medical or clinical notations to extract meaning for themselves as explained by Quote 5. Of the 8 participants only 2 were Danish natives. The other 6 participants who were not native speakers, reported difficulties using Sundhed because of the barriers with the Danish language in medicine contexts, and a lack of options for customizing the language experience as participant 6 mentions in Quote 6. 


\section{Attitudes Towards Services and Platforms Using Combined Data Sources}

Half of the participants saw opportunities in predictive analytics as a service that would combine data from a variety of available sources. This service would increase the awareness of personal health by showing how mental and physical health develops over time. The participants thought that preferably this service should be able to detect and report on patterns of mental and physical health and wellbeing through statistics or customization options as discussed in Quote 7. The majority of participants expressed the need for a service that would interface different data sources to incorporate the use of their PHD. This service, which if not part of the public sector, would have to be regulated heavily by legal governing bodies.

Despite the wish of the aforementioned participants however, a couple of the participants expressed negative attitudes towards an idea of combining personal data sources, especially if it requires central data storage. Concerns of data security around personal data stored across different data sources was strongly emphasized. Also, the participants expressed hesitation around the management of the data collection if combining the data sources required the individuals to manually consent, assemble, and finance their personal data collection. In addition, apprehensions of data handling by other parties whom were not given exclusive permission to use the data by the data owners, was identified as a point of contention for participants.

\section{E. The Vision for Data Format and Visualizations}

Participants reported that they found a variety of different data formats such as .xlsx and PDF. They thought that handling the data in different contexts would be dependent on how the data could be used afterwards, for example processing or having an overview of data should correspond to different data formats.

As illustrated in Quote 8, the majority of participants suggested that visualizations, such as bar and pie charts, or images could be used for visualizing PHD. Participants emphasized that visualizations will be a valuable tool when dealing with large amounts of data, connecting the usefulness of PHD to how their information is being visualized. Some participants suggested that visualizations of PHD will be more effective with the addition of customizable components, such as parameter declarations that will enable direct interaction with the data.

TABLE II. SUMMARY OF SUBTHEMES AND QUOTES

\begin{tabular}{|c|c|c|c|}
\hline Quote\# & Participant\# & Subtheme & Quote \\
\hline Quote 1 & \# 7 & Access Protection & $\begin{array}{l}\text { "[...] and they only asked for my name and CPR number, and I was really disappointed about } \\
\text { that. Because a CPR number is often treated as a secret in the public system..." }\end{array}$ \\
\hline Quote 2 & \#2 & Access Protection & $\begin{array}{l}\text { "I wouldn't mind as long as this is not taken by [a] third party and used [in] a way that is not } \\
\text { correct, whatever that could be." }\end{array}$ \\
\hline Quote 3 & \#2 & Accessibility Challenges & $\begin{array}{l}\text { "One challenge would be in Sundhed.dk, as I've mentioned before I had [tried] } 3 \text { links to find } \\
\text { my appointments and one was working..." }\end{array}$ \\
\hline Quote 4 & $\# 5$ & Accessibility Challenges & $\begin{array}{l}\text { "Through Google for everything, so first I've made a little brainstorm, ok, where can I find } \\
\text { [the] doctor... just Google it." }\end{array}$ \\
\hline Quote 5 & \#8 & $\begin{array}{l}\text { Perceived Value of } \\
\text { Accessed Personal Data }\end{array}$ & $\begin{array}{l}\text { "Because everything is in doctor terminology so you need to take a little step back and think } \\
\text { what it actually means" }\end{array}$ \\
\hline Quote 6 & \#6 & $\begin{array}{l}\text { Perceived Value of } \\
\text { Accessed Personal Data }\end{array}$ & $\begin{array}{l}\text { "... although I speak Danish, there are things especially like medical things the way that are } \\
\text { written like that you can't' really understand." }\end{array}$ \\
\hline Quote 7 & \#3 & $\begin{array}{l}\text { Attitudes Towards Services } \\
\text { and Platforms Using } \\
\text { Combined Data Sources }\end{array}$ & $\begin{array}{l}\text { "I mean that would be nice actually you have your patterns or whatever [...] predictions! So, } \\
\text { you change bad habits or they say this is good, so you are more aware about that, that would } \\
\text { be perfect." }\end{array}$ \\
\hline Quote 8 & \#6 & $\begin{array}{l}\text { The Vision for Data } \\
\text { Format and Visualizations }\end{array}$ & "I am a very visual person so I would like to see some graphs..." \\
\hline
\end{tabular}

\section{DISCUSSION}

The scope of the study was to explore how accessible personal health related data is and how valuable the participants found the data describing their health. The results of this study revealed that participants found it to be relatively easy to access to their PHD; contrary to the results of the [11] study. In regards to the format of personal data, [11] and [12] studies concluded that their participants had limited access to health data through digital services or platforms, reporting that they had to send requests via mail to various organizations and to receive personal data in paper format. Our participants were able to access most of their data through digital interfaces. This could be explained as a couple of years have lapsed, there has been advancements in digitalization of health data, or that in Denmark, organizations are offering better access to digital data in general.
This study delineates that perceived value is connected to the context of individual participants. Despite the fact that participants did in fact have access to their PHD through digital services and platforms in Denmark, the perceived value of these experiences is considered to be low due to poor user experience. When users were engaged with such platforms as Sundhed, there was a blatant lack of contextual understanding of medical jargon because the participants are not medical experts. However, Sundhed provides a medical dictionary that is available in the platform for the sole purpose of translating medical terminology into layman's terms. Regardless to the existence of this dictionary, poor user interface design in Sundhed left the participants' experience as a negative one as the dictionary was not acknowledged. In the future, the experience of a user should be a positive one, and thus considerations for more intuitive designs in user interfaces is important. 
Complementary to the idea of looking towards the future in Denmark's digital health arena, is providing perceived value through the use of personalization. The accessibility of the PHD in Denmark is now not as important to consider as the future value that can be derived from the experience of engaging with PHD. The results of the present study suggest that individuals are not ready to utilize their PHD because on the one hand they do not know where to find services and platforms, and on the other, there is a lack of tools available that enables engagement in a way that residents can derive contextual value from. Services and platforms in Denmark should strive for supporting multi-format integration and establishing a format standardization that services and platforms must adhere to when providing access to residents.

The attitude towards an idea of integration of data sources was appealing to the participants if that would offer them visualization and customization potential of their PHD, allowing each individual to create their own context for what they deemed to be valuable. Visualizations are considered to be a medium that could enable individuals to identify emerging trends faster, to interact with real-time data and to grasp more information in an easier manner. Interestingly, an important issue the participants addressed in combining different data sources is a need to control who can access their data. This concern of access control of personal data is reflected throughout previous research for centralizing patient data [16] and future services and platforms in Denmark should be mindful of this attitude.

Our study design addressed the limitation of recruiting researchers reported by study [11], by sampling from the general healthy population of Denmark. However, eight persons should not be considered a large enough sample size to generalize about the population of Denmark.

One of the major challenges that this research confronts is how to create value from data. The present study explored participants' experience related to accessing their PHD and the perceived value of data for future self-care management. Our results suggest that new technical components such as visualizations or features that support personalization of PHD could create value for individuals by supporting their contextual access-based needs. In the future, an individual's active participation in health care systems is crucial for involving the individual in their personal health and wellness.

\section{CONCLUSION}

The scope of this study was to explore how accessible and valuable PHD are for the general healthy population in Denmark. The aim being to generate knowledge relevant for the healthcare services and platforms to increase value for all stakeholders. Our study points out that although PHD are accessible through digital platforms and services in Denmark, there is a need for personalization, standardization of formats and improvement of users' experience all towards increasing the perceived value. With the increasing human and data mobility in Europe, future research should also explore methods that would address the needs of multilingualism.

\section{ACKNOWLEDGMENT}

C.G. Author has received funding from the European Union's Horizon 2020 research and innovation programme -
Marie Sklodowska-Curie Actions grant agreement no. 676201 - CHESS - Connected Health Early Stage Researcher Support.

\section{ETHICS APPROVAL AND CONSENT TO PARTICIPATE}

The ethics committee of ITU follows the principles of the Danish Code of Conduct for Research Integrity, which states that ethical approval is not required for the type of study presented here. The participants signed an informed consent form before the study and were aware of their right to withdraw from the study at any time.

\section{REFERENCES}

[1] Sellen, A., Rogers, Y., Harper, R. and Rodden, T., 2009. Reflecting human values in the digital age. Communications of the ACM, 52(3), pp.58-66.

[2] Eagle, N. and Pentland, A.S., 2006. Reality mining: sensing complex social systems. Personal and ubiquitous computing, 10(4), pp.255268.

[3] Zhang, D., Guo, B., Li, B. and Yu, Z., 2010. Extracting social and community intelligence from digital footprints: an emerging research area. Ubiquitous Intelligence and Computing, pp.4-18.

[4] Malhotra, A., Totti, L., Meira Jr, W., Kumaraguru, P. and Almeida, V., 2012, August. Studying user footprints in different online social networks. Proceedings of the 2012 IEEE/ACM International Conference on Advances in Social Networks Analysis and Mining, ASONAM 2012, (pp. 1065-1070).

[5] Google Now, accessed December 2017, https://goo.gl/ti7UXg.

[6] Siri, accessed December 2017, https://goo.gl/uKi1NC.

[7] Raghupathi, W. and Raghupathi, V., 2014. Big data analytics in healthcare: promise and potential. Health information science and systems, 2(1), p.3.

[8] Li, I., Dey, A. and Forlizzi, J., 2010, April. A stage-based model of personal informatics systems. In Proceedings of the SIGCHI Conference on Human Factors in Computing Systems, (pp. 557-566).

[9] Tang, P.C., Ash, J.S., Bates, D.W., Overhage, J.M. and Sands, D.Z., 2006. Personal health records: definitions, benefits, and strategies for overcoming barriers to adoption. Journal of the American Medical Informatics Association, 13(2), pp.121-126.

[10] Pagliari, C., Detmer, D. and Singleton, P., 2007. Potential of electronic personal health records. BMJ: British Medical Journal, 335(7615), p.330.

[11] Harjumaa, M., Saraniemi, S., Pekkarinen, S., Lappi, M., Similä, H. and Isomursu, M., 2016. Feasibility of digital footprint data for health analytics and services: an explorative pilot study. BMC medical informatics and decision making, 16(1), p.139.

[12] Gencoglu, O., Similä, H., Honko, H. and Isomursu, M., 2015, August. Collecting a citizen's digital footprint for health data mining. In Engineering in Medicine and Biology Society (EMBC), 2015 37th Annual International Conference of the IEEE (pp. 7626-7629).

[13] Kujala, S. and Väänänen-Vainio-Mattila, K., 2009. Value of information systems and products: Understanding the users' perspective and values. Journal of Information Technology Theory and Application (JITTA), 9(4), p.4.

[14] Levesque, J., Harris, M. and Russell, G. (2013). Patient-centred access to health care: conceptualising access at the interface of health systems and populations. International Journal for Equity in Health, 12(1), p.18.

[15] Datatilsynet, accessed December 2017, https://goo.gl/cQuYJp.

[16] Angst, C.M. and Agarwal, R., 2009. Adoption of electronic health records in the presence of privacy concerns: The elaboration likelihood model and individual persuasion. MIS quarterly, 33(2), pp.339-370. 